\title{
Clinical evaluation of the role of ceftaroline in the management of community acquired bacterial pneumonia
}

This article was published in the following Dove Press journal:

Infection and Drug Resistance

31 January 2012

Number of times this article has been viewed

\author{
Diego J Maselli' \\ Juan F Fernandez' \\ Christine Y Whong ${ }^{2}$ \\ Kelly Echevarriaa ${ }^{1,3}$ \\ Anoop M Nambiar ${ }^{1,3}$ \\ Antonio Anzueto 1,3 \\ Marcos I Restrepo 1,3,4 \\ 'University of Texas Health Science \\ Center, San Antonio, Texas, ${ }^{2}$ Memorial \\ Hermann - Texas Medical Center, \\ Houston, TX, ${ }^{3}$ South Texas Veterans \\ Health Care System Audie I Murphy \\ Division, San Antonio, TX, ${ }^{4}$ Veterans \\ Evidence Research Dissemination and \\ Implementation Center (VERDICT), \\ San Antonio, TX, USA
}

\begin{abstract}
Ceftaroline fosamil (ceftaroline) was recently approved for the treatment of community-acquired pneumonia (CAP) and complicated skin infections. This newly developed cephalosporin possesses a broad spectrum of activity against gram-positive and gramnegative bacteria. Most importantly, ceftaroline demonstrates potent in vitro antimicrobial activity against multi-drug resistant Streptococcus pneumoniae and methicillin-resistant strains of Staphylococcus aureus. In two Phase III, double-blinded, randomized, prospective trials (FOCUS 1 and FOCUS 2), ceftaroline was shown to be non-inferior to ceftriaxone for the treatment of CAP in hospitalized patients. Ceftaroline exhibits low resistance rates and a safety profile similar to that of other cephalosporins. In this review, we will evaluate the pharmacological characteristics, safety, antimicrobial properties, and efficacy of ceftaroline and its applications in the treatment of CAP.
\end{abstract}

Keywords: s. pneumoniae, s. aureus, cephalosporins, pneumonia, ceftaroline, community acquired pneumonia

\section{Introduction}

The ideal antibiotic for the treatment of community-acquired pneumonia (CAP) should have the following characteristics: (a) a spectrum of activity that covers the majority of pathogens associated with this infection; (b) documented clinical efficacy and safety in a variety of patient populations; and (c) cost-effectiveness. Current guidelines recommend stratifying patients into groups depending on the presence of specific risk factors and evaluating health care utilization history to select appropriate empirical antimicrobial therapy. ${ }^{1}$ The implementation of these guidelines has greatly increased the rate of treatment success for CAP., ${ }^{2,3}$ Despite this, treatment failures continue to exist and the need for more effective therapies is a consequence of two main issues: the emergence of antimicrobial resistance and newly emerging pathogens causing CAP.

The rise in the detection of multi-drug resistant Streptococcus pneumoniae (S. pneumoniae) (MDRSP) has caused significant concern. ${ }^{4,5}$ This pathogen displays elevated minimum inhibitory concentrations (MIC) for penicillin and cephalosporins, and often demonstrates cross resistance with other classes, including macrolides, tetracycline and trimethoprim/sulfamethoxazole; although the fluoroquinolone class is relatively spared. ${ }^{6}$ There is controversy regarding how in vitro resistance translates into clinical outcomes. Studies evaluating the mortality of patients affected by MDRSP compared to more susceptible strains of $S$. pneumoniae have shown conflicting results. ${ }^{7-10}$ However, some of these studies were limited by confounding factors including age, comorbidities, and severity of illness. ${ }^{8}$ Nevertheless, there are reports that patients with MDRSP
Correspondence: Marcos I Restrepo VERDICT (I IC6), South Texas Veterans Health Care System ALMD, 7400 Merton Minter Boulevard San Antonio, TX 78229, USA

Tel + I 2106175300 extension I54I3

Fax + I 2105674423

Email restrepom@uthscsa.edu 
may have more complications, longer hospital lengths of stay, and greater therapeutic failures. ${ }^{6,11,12}$

Staphylococcus aureus (S. aureus) is a dynamic pathogen that continues to pose a great challenge to clinicians treating patients with CAP and other infections. Methicillin-resistant strains of $S$. aureus (MRSA) infections cause significant mortality and contribute to increased health care costs. ${ }^{13-15}$ Most worrisome are data demonstrating a rise in CAP due to MRSA. ${ }^{16-19}$ Community-associated MRSA characteristically belongs to the USA-300 pulse-field electrophoresis type, containing the Panton-Valentine leukocidin gene, and is an important cause of necrotizing CAP. ${ }^{20,21}$ Compared to other sites of infection, CAP caused by MRSA of the USA-300 type is associated with worse clinical outcomes. ${ }^{22}$ Due to these emerging trends of resistance and the need to treat a wider range of patient populations, newer therapies should be explored for the treatment of MRSA, and other resistant gram-positive bacteria. ${ }^{23}$

The USA Federal Drug Administration (FDA) has recently approved ceftaroline fosamil (TEFLARO ${ }^{\mathrm{TM}}$, Forest Pharmaceuticals Inc, St Louis, MO) for the treatment of CAP and complicated skin infections. This antibiotic possesses a broad spectrum of activity against gram-positive and gram-negative bacteria. Most importantly, ceftaroline has antimicrobial activity against MDRSP and MRSA. We will review the pharmacological characteristics, safety, antimicrobial properties, and effectiveness of ceftaroline and its applications in the treatment of CAP patients.

\section{Mechanisms of action}

Ceftaroline is a fifth-generation cephalosporin with a mechanism of action similar to that of other commercially available $\beta$-lactams. ${ }^{24-26}$ It binds to penicillin binding proteins (PBPs) and prevents the synthesis of peptidoglycan, an essential component in bacterial cell walls. ${ }^{24-26}$ The drug's activity against $S$. aureus and MRSA is due to its high affinity for PBP1-3 and PBP2a. ${ }^{24-26}$ Additionally, ceftaroline binds to PBP3, PBP1A, PBP2X, PBP1B, and PBP2A/B, which are primary targets for $S$. pneumoniae, including resistant strains. ${ }^{24-26}$

\section{Microbiologic activity}

Ceftaroline has demonstrated activity against a broad spectrum of gram-positive pathogens (Table 1). Several studies evaluating the in vitro activity of ceftaroline have been carried out (Table 2). ${ }^{27-33}$ A surveillance study of 6,496 CAP pathogens compared ceftaroline to comparator agents. ${ }^{32}$ Ceftaroline was found to be eight-fold more active against
Table I Spectrum of microbiological coverage

\begin{tabular}{ll}
\hline Gram-positive bacteria & Gram-negative bacteria \\
\hline - Staphylococcus aureus (MSSA and MRSA) & - Klebsiella pneumoniae \\
- Streptococcus pyogenes & - Klebsiella oxytoca \\
- Streptococcus agalactiae & - Escherichia coli \\
- Streptococcus pneumoniae & - Citrobacter koseri \\
- Streptococcus dysgalactiae & - Citrobacter freundii \\
& - Enterobacter cloacae \\
& - Enterobacter aerogenes \\
& - Haemophilus influenzae \\
& - Haemophilus parainfluenzae \\
& - Proteus mirabilis \\
& - Moraxella catarrhalis \\
\hline
\end{tabular}

$\overline{\text { Abbreviations: MRSA, methicillin-resistant Staphylococcus aureus; MSSA, methicillin- }}$ susceptible Staphylococcus aureus.

1,340 S. pneumoniae strains than ceftriaxone. Furthermore, ceftaroline exhibited excellent activity against MDRSP. ${ }^{32,34,35}$ Another group found ceftaroline to be the most active agent against 120 strains of cefotaxime-resistant S. pneumoniae. ${ }^{36}$ Additionally, ceftaroline was noted to have potent activity against strains with defined PBPs and murM mutations known to confer resistance to $\beta$-lactams. ${ }^{36}$ Similar results were evidenced in a susceptibility study of 891 strains of $S$. pneumoniae isolated in the United States, which compared ceftaroline with current available antibiotic therapies. Again, ceftaroline was the most effective agent tested and displayed a high level of activity against the subset of strains considered to be resistant to penicillin, macrolides, lincosamides, trimethoprim-sulfamethoxazole, and quinolones. ${ }^{31}$ Likewise, in another study, ceftaroline demonstrated very high potency against 584 strains of Haemophilus influenzae and 377 strains of Moraxella catarrhalis - a degree of activity comparable to ceftriaxone. ${ }^{32}$ The characteristics of ceftaroline were tested in vivo in an experimental pneumonia rabbit model where subjects were inoculated with different strains of S. pneumoniae. Ceftaroline was similar to ceftriaxone in eradicating the infection in subjects infected with penicillin susceptible $S$. pneumoniae strains; but more importantly, ceftaroline was superior in the treatment of MDRSP strains. ${ }^{37}$

Ceftaroline is the first cephalosporin approved to have in vitro activity against methicillin-resistant $S$. aureus (MRSA), a characteristic that may prove to be particularly useful if this pathogen continues to increase in frequency. Large series of isolates have demonstrated bactericidal activity against coagulase-negative staphylococci as well as methicillin-susceptible and methicillin-resistant S. aureus. ${ }^{29,32}$ Furthermore, ceftaroline has exhibited potent activity against vancomycin-intermediate $S$. aureus (VISA), vancomycin-resistant $S$. aureus (VRSA), linezolid-resistant 
Table 2 Susceptibility for ceftaroline in in vitro studies

\begin{tabular}{|c|c|c|c|c|c|c|c|}
\hline \multirow[t]{2}{*}{ Pathogens } & \multicolumn{7}{|c|}{ In vitro studies - MIC range $(\mu \mathrm{g} / \mathrm{mL})$} \\
\hline & Sader ${ }^{27}$ & $\mathbf{G e}^{28}$ & Brown ${ }^{29}$ & Saravolatz ${ }^{30}$ & Jacobs $^{31}$ & Jones 32 & Kaushik $^{33}$ \\
\hline S. aureus & & & 0.25 to 2 & & & $\leq 0.12$ to 2 & \\
\hline MRSA & 0.12 to 2 & 0.12 to 2 & 0.5 to I & & & $\leq 0.25$ to 2 & 0.25 to 2 \\
\hline MSSA & 0.03 to 0.5 & $\leq 0.03$ to I & 0.25 to 0.5 & & & $\leq 0.1$ to 0.5 & $\leq 0.008$ to $\mathrm{I}$ \\
\hline CA-MRSA & & & & $\leq 0.25 \mathrm{I}$ & & & \\
\hline VISA/hVISA & 0.25 to 4 & & 0.25 to 2 & $\leq 0.25 \mathrm{I}$ & & & 0.25 to 4 \\
\hline VRSA & & & 0.5 to $\mathrm{I}$ & $\leq 0.121$ & & & \\
\hline Streptococcus pneumoniae & & & 0.015 to 0.5 & & $\leq 0.008$ to 0.5 & $\leq 0.008$ to 0.5 & \\
\hline Streptococcus pneumoniae & 0.06 to 0.5 & $\leq 0.008$ to 0.5 & 0.015 to 0.5 & & $\leq 0.006$ to 0.5 & & $\leq 0.008$ to 0.5 \\
\hline \multicolumn{8}{|l|}{$\mathrm{PCN}$ resistant } \\
\hline Streptococcus pneumoniae & $\leq 0.016$ to 0.06 & $\leq 0.008$ to 0.12 & 0.015 to 0.12 & & & & $\leq 0.008$ to 0.12 \\
\hline \multicolumn{8}{|l|}{ PCN susceptible } \\
\hline Streptococcus pyogenes & & $\leq 0.008$ to 0.03 & 0.015 to 0.5 & & & & \\
\hline \multicolumn{8}{|l|}{ Macrolide resistant } \\
\hline Moraxella catarrhalis & $\leq 0.016$ to 012 & $\leq 0.03$ to 0.5 & 0.015 to 1 & & & $\leq 0.008$ to $\mathrm{I}$ & $\leq 0.008$ to 0.5 \\
\hline Haemophilus influenzae & $\leq 0.016$ to 0.25 & $\leq 0.008$ to 2 & 0.015 to 0.25 & & & $\leq 0.008$ to 0.12 & $\leq 0.008$ to 0.25 \\
\hline Klebsiella pneumoniae & 0.03 to 4 & $\leq 0.03$ to $>16$ & 0.03 to 1 & & & $\leq 0.008$ to $>16$ & $\leq 0.03$ to 165 \\
\hline Escherichia coli & $\leq 0.016$ to 0.025 & $\leq 0.03$ to $>16$ & 0.015 to 16 & & & $\leq 0.015$ to $>16$ & 0.5 to $>16$ \\
\hline Enterobacter cloacae & 0.03 to $>32$ & $\leq 0.03$ to $>16$ & 0.06 to 2 & & & $\leq 0.015$ to $>16$ & $\leq 0.03$ to $>16$ \\
\hline Pseudomonas aeruginosa & 4 to $>32$ & & 0.5 to 32 & & & & I to $>128$ \\
\hline
\end{tabular}

Abbreviations: MIC, minimum inhibitory concentration; MRSA, methicillin-resistant Staphylococcus aureus; MSSA, methicillin-susceptible Staphylococcus aureus; CA-MRSA, community acquired methicillin-resistant Staphylococcus aureus; VISA/hVISA, vancomycin-intermediate S. aureus/hetero-resistant VISA; VRSA, vancomycinresistant S. aureus; PCN, penicillin.

S. aureus (LRSA), and daptomycin-nonsusceptible S. aureus (DNSSA). ${ }^{30,38}$ The activity against DNSSA was confirmed in a study that tested ceftaroline against four different strains. Ceftaroline showed sustained bactericidal activity against three of the strains and a sustained reduction in the bacterial counts with respect to the fourth. ${ }^{39}$

Ceftaroline has similar microbial coverage as other thirdgeneration cephalosporins for gram-negative microorganisms (Table 1). The potency of ceftaroline is comparable with ceftriaxone, ceftazidime, and piperacillin/tazobactam for Escherichia coli (E. coli), Klebsiella pneumoniae, and Enterobacter cloacae. ${ }^{32}$ However, it is important to note that ceftaroline, in a similar fashion to third-generation cephalosporins, does not have significant in vitro activity against extended-spectrum beta-lactamase (ESBL) producing organisms, Pseudomonas sp., or atypical microorganisms.

\section{Pharmacokinetics}

The absorption of ceftaroline has been analyzed in healthy adults. The prodrug ceftaroline fosamil acetate (CFA) and inactivated ceftaroline metabolites displayed linear-dose kinetics. ${ }^{33}$ Additionally, ceftaroline's concentrations were not associated to the duration of the dosing interval. The drug's pharmacokinetic analysis is compatible with a twocompartmental model with zero order input and first order elimination. ${ }^{40}$ The volume of distribution of ceftaroline is
$28.3 \mathrm{~L}(0.37 \mathrm{~L} / \mathrm{kg}$; range $0.31-0.45 \mathrm{~L} / \mathrm{kg})$ and the drug does not significantly bind to serum proteins, with less than $20 \%$ being protein-bound. CFA does not have any penetration into erythrocytes. ${ }^{33}$

Ceftaroline fosamil acetate is rapidly metabolized by phosphatases, which convert it to ceftaroline after intravenous administration. The active drug then undergoes further conversion by hydrolysis into the inactive ceftaroline-M-1. The average half-life of ceftaroline and ceftaroline M-1 is 2.6 hours and 4.5 hours, respectively. ${ }^{41}$ Of the ceftaroline metabolites, ceftaroline is not detected in the urine, while approximately $50 \%$ of the dose is excreted as active drug with a small portion (average 7\%) excreted as ceftarolineM-1. ${ }^{41}$ Ceftaroline has only minimal enteric elimination. In patients with mild renal impairment (creatinine clearance [CrCl] of $50-80 \mathrm{~mL} / \mathrm{min}$ ), the area under the curve (AUC) was $25 \%$ higher and the half-life $14 \%$ longer. In patients with moderate renal impairment $(\mathrm{CrCl} 30-50 \mathrm{~mL} / \mathrm{min})$, the AUC was $50 \%$ higher. Because of these characteristics, ceftaroline should be used with caution in patients with moderate to severe renal impairment, and dosing adjustments according to renal function are advised. ${ }^{42}$

The dosing recommendation for intravenous dosing is as follows depending on the $\mathrm{CrCl}$ : (1) higher than $50 \mathrm{~mL} / \mathrm{min}$ : no adjustment (600 mg every 12 hours); (2) 30-50 mL/min: $400 \mathrm{mg}$ every 12 hours; (3) 15-30 mL/min: 
$300 \mathrm{mg}$ every 12 hours; (4) for patients on dialysis: $200 \mathrm{mg}$ every 12 hours. ${ }^{41,43}$ Animal models have shown a favorable pharmacokinetic profile with intramuscular administration comparable to intravenous dosing. ${ }^{44,45}$ The absolute bioavailability after an intramuscular dose was equivalent to an intravenous dose. ${ }^{43}$

\section{Pharmacodynamics}

Ceftaroline exhibits time-dependent killing. As such, the amount of time that the serum concentration remains above the minimum inhibitory concentration (MIC); ( $\% \mathrm{~T}>\mathrm{MIC})$ represents the main pharmacodynamic predictor of efficacy. Pharmacodynamic evaluations have been performed in murine thigh and lung infection models. ${ }^{46}$ The results from this study demonstrated a minimal post-antibiotic effect ranging from 0.33 hours to 7.2 hours for $S$. pneumoniae, E. coli, and $S$. aureus. ${ }^{46}$ Additionally, ceftaroline was found to be bacteriostatic for staphylococci and gram-negative bacilli when free drug concentration exceeded the MIC for $30 \%$ and $40 \%$ of the dosing interval, respectively. ${ }^{46}$ On the other hand, bactericidal activity for staphylococci and gramnegative bacilli with ceftaroline occurred when $\% \mathrm{~T}>\mathrm{MIC}$ was $50 \%$ and $60 \%$, respectively. ${ }^{46}$

\section{Clinical use and efficacy in CAP}

The efficacy and safety of ceftaroline for the treatment of CAP was evaluated in the FOCUS (ceFtarOline Communityacquired pneUmonia) trial against ceftriaxone in hospitalized patients with CAP. This study included two similar, Phase III, double-blinded, randomized, multinational, prospective trial designs (FOCUS 1 and FOCUS 2$).{ }^{47}$ The primary objective of these studies was to determine non-inferiority in clinical cure rates of ceftaroline compared with ceftriaxone in the clinically evaluable and modified intent-to-treat efficacy populations. Clinical cure was defined as resolution of all signs and symptoms of pneumonia or improvement such that no further antimicrobial therapy was necessary. ${ }^{47}$ Patients were also required to have absence of fever for 24 consecutive hours with signs and symptoms of CAP returning to baseline levels. ${ }^{47}$

The study design for both trials involved randomization of patients with CAP based severity using the Pneumonia Outcomes Research Team (PORT) score of III or IV (patients that required an admission to the hospital for administration of intravenous antibiotics) to receive either $600 \mathrm{mg}$ intravenously of ceftaroline every 12 hours or one gram intravenously of ceftriaxone daily for 5-7 days. Importantly, the studies excluded patients who were not admitted to the hospital (PORT I and II) or directly to the intensive care unit (ICU) (PORT V). Other notable exclusion criteria were patients with severe renal impairment $(\mathrm{CrCl} \leq 30 \mathrm{~mL} / \mathrm{min})$, risk factors for hospitalacquired infections, known or suspected infections with atypical microorganisms, risk factors or positive cultures for MRSA, and immunosuppression. The sole difference between these two studies was that the patients enrolled in FOCUS 1 received two doses of clarithromycin on day one. This additional treatment was required to enable enrollment in North America, where macrolide therapy is recommended, but to limit potential confounding of study drug treatment effect, it was only given during the first 24 hours of treatment.

An integrated analysis of both trials included a total of 1,228 patients (ceftaroline, $\mathrm{n}=614$ versus ceftriaxone, $\mathrm{n}=614$ ). Baseline characteristics were similar between the groups. The study groups had the following similar baseline characteristics: age distribution, race, gender, comorbid conditions, PORT scores, white-blood cell counts, bacteremia, and immature band counts. The primary outcomes demonstrated that of the clinically evaluable patients treated with ceftaroline, $84.3 \%$ achieved a clinical cure, compared with $77.7 \%$ of patients treated with ceftriaxone $(95 \% \mathrm{CI}$, $1.6 \%-11.8 \%$ ). In the modified intent-to-treat efficacy population, clinical cure was achieved in $82.6 \%$ of the patients treated with ceftaroline, compared to $76.6 \%$ treated with ceftriaxone (95\% CI, 1.4\%-10.7\%). The adverse effects and tolerability of the medications were similar in both groups. There were 27 reported deaths during the study; 15 (2.4\%) in the ceftaroline group and $12(2.0 \%)$ in the ceftriaxone group (Table 3$){ }^{47}$

The most frequent isolated pathogen was $S$. pneumoniae, with a combined prevalence of $33.6 \%$ (122 isolates), which is consistent with the epidemiology of CAP. The clinical cure rates for $S$. pneumoniae were $85.7 \%$ (54 of 63 patients) for ceftaroline and $69.5 \%$ (41 of 59 patients) for ceftriaxone. Even though the total number of patients treated for MDRSP CAP was low $(n=13)$, ceftaroline exhibited a higher rate of clinical cure $(4 / 4,100 \%)$ compared to ceftriaxone $(2 / 9,22 \%)$.

Staphylococcus aureus was the second most common pathogen isolated with an incidence of $14.3 \%$ (52 isolates). The clinical cure rates for $S$. aureus were $72.0 \%$ (18 of 25 patients) for ceftaroline and 55.6\% (15 of 27 patients) for ceftriaxone. Out of the $S$. aureus isolates, only two were MRSA, and both were in the ceftriaxone study arm. The low 
Table 3 Clinical cure rates comparing ceftaroline against ceftriaxone in CAP patients enrolled in the FOCUS I and FOCUS 2 studies

\begin{tabular}{|c|c|c|c|c|c|c|}
\hline \multirow[t]{2}{*}{ Test of cure } & \multicolumn{2}{|l|}{ FOCUS I } & \multicolumn{2}{|l|}{ FOCUS 2} & \multicolumn{2}{|c|}{ Integrated FOCUS I and 2} \\
\hline & Ceftaroline & Ceftriaxone & Ceftaroline & Ceftriaxone & Ceftaroline & Ceftriaxone \\
\hline & \multicolumn{6}{|c|}{ Primary outcomes $n / \mathbf{N}(\%)$} \\
\hline Clinical evaluable & $194 / 224(86.6)^{*}$ & 244/29I (83.8) & 193/235 (82) & $166 / 215(77)$ & $387 / 459(84)^{*}$ & $349 / 449$ (77) \\
\hline \multirow[t]{2}{*}{ Modified intent to treat efficacy } & 244/29I (83) & $23 / 300(77)$ & $235 / 289(81)$ & $206 / 273(75)$ & $479 / 580(82)^{*}$ & $439 / 573(76)$ \\
\hline & \multicolumn{6}{|c|}{ Secondary outcomes } \\
\hline Microbiologically evaluable & $62 / 69(89)^{*}$ & $54 / 71(76)$ & 69/85 (8I) & $57 / 76(75)$ & $13|/| 54(85)^{*}$ & III//47 (75) \\
\hline Streptococcus pneumoniae & $24 / 27(88.9)$ & $20 / 30(66)$ & $35 / 42(83.3)$ & $28 / 40(70)$ & $59 / 69(85.5)$ & $48 / 70(68)$ \\
\hline Streptococcus pneumoniae & $2 / 2(100)$ & $0 / 1(0)$ & $2 / 2(100)$ & $2 / 8(25)$ & $4 / 4(100)$ & $2 / 9(22)$ \\
\hline \multicolumn{7}{|l|}{$\mathrm{PCN}$ resistant } \\
\hline Staphylococcus aureus & $8 / 10(80)$ & $9 / 14(64)$ & $10 / 15(66.7)$ & $9 / 16(56)$ & $18 / 25(72)$ & $18 / 30(60)$ \\
\hline Haemophilus influenzae & $4 / 5(80)$ & $7 / 10(70)$ & 13/15 (86) & I3/14 (92) & $17 / 20(85)$ & 20/24 (83) \\
\hline Klebsiella pneumoniae & $7 / 8(87)$ & $3 / 5(60)$ & $7 / 7(100)$ & $7 / 8(87)$ & $14 / 15$ (93) & $10 / 13(76)$ \\
\hline Escherichia coli & $8 / 8(100)$ & 5/7 (7I) & $2 / 4(50)$ & $4 / 6(66)$ & $10 / 12(83)$ & $9 / 13(69)$ \\
\hline $\begin{array}{l}\text { Microbiological modified } \\
\text { intent to treat efficacy }\end{array}$ & 66/75 (88)* & $60 / 80(75)$ & $72 / 90(80)$ & $66 / 88(75)$ & I38/165 (83) & I26/I68 (75) \\
\hline PORT risk class III & $136 / 150(90)^{*}$ & II3/I42 (79) & $113 / 137$ (82) & $104 / 132(78)$ & $249 / 287(86)^{*}$ & $217 / 274(79)$ \\
\hline PORT risk class IV & $58 / 74(78)$ & $70 / 92(76)$ & $80 / 98(8 \mathrm{I})$ & $62 / 83(74)$ & I38/I $72(80)$ & $132 / 175(75)$ \\
\hline End of therapy & \multicolumn{4}{|c|}{ Secondary outcomes } & & \\
\hline Clinical evaluable & $197 / 224(87)^{*}$ & I88/234 (80) & 102/235 (86) & $172 / 215(80)$ & & \\
\hline
\end{tabular}

Note: $* P<0.05$ when comparing ceftaroline versus ceftriax.

Abbreviations: PCN, penicillin; PORT, Pneumonia Outcomes Research Team.

prevalence of MRSA is likely due to both a relative low frequency of occurrence of this pathogen causing CAP and the exclusion criteria of the FOCUS 1 and FOCUS 2 studies. ${ }^{48}$

Based on the above findings, the authors concluded that ceftaroline was clinically non-inferior to ceftriaxone. Furthermore, the integrated analysis demonstrated a favorable trend towards ceftaroline improving clinical cure rates reaching statistical significance. These outcomes strongly support the efficacy and safety of ceftaroline treatment in hospitalized, non-ICU patients with CAP, but leave unanswered questions regarding efficacy in other populations. Given the exclusion criteria of these studies, data are lacking regarding the efficacy of ceftaroline for CAP treatment in patients that are immunosuppressed, require ICU admission, or have risk factors for MRSA and other hospital-acquired infections. Importantly, ceftaroline showed clinical efficacy for the treatment of MDRSP, but larger studies are needed to confirm these findings. Currently, the USA Federal Drug Administration (FDA) has approved ceftaroline as an option for the treatment of CAP.

\section{Safety}

The frequency of diarrhea, nausea, vomiting, constipation, transaminitis, hypokalemia, rash, and phlebitis was similar in patients that received ceftaroline compared to treatment regimens with vancomycin plus aztreonam (skin and skin structure studies) or ceftriaxone..$^{47,49}$ Developmental toxicity studies performed in rats that received ceftaroline at a dose eight times greater than the human dose did not demonstrate maternal toxicity or effects on the fetus. Currently, there are no adequate trials evaluating the use of ceftaroline in pregnant women, and so it should be used with caution and then only when the potential benefits outweigh potential risks to the fetus. It has not been determined if ceftaroline is excreted in the human milk and caution should be exercised when administering this medication to a nursing woman. Long-term studies evaluating carcinogenesis, mutagenesis, and effects on fertility have not been performed on ceftaroline.

The ecological impact of ceftaroline in human intestinal microflora has been evaluated after healthy subjects received $600 \mathrm{mg}$ intravenously (IV) every 12 hours for 7 days. ${ }^{50} \mathrm{In}$ this study, there was no significant impact on the numbers of resistant E. coli, Bacteroides sp., Enterococcus sp., or Candida albicans strains. More importantly, no new colonizing aerobic or anaerobic bacteria resistant to ceftaroline (MIC $>4 \mathrm{mg} / \mathrm{L}$ ) were detected. Additionally, the incidence of Clostridium difficile infection with ceftaroline was similar to other cephalosporins.

\section{Comparative advantages}

As compared to other clinically available agents, ceftaroline exhibits low resistance rates and possesses a broader 
spectrum activity against common pathogens implicated in CAP. Furthermore, ceftaroline's safety profile is similar to that of current antimicrobials utilized to treat these infections. The most common adverse effects reported with ceftaroline were diarrhea, nausea and vomiting, but serious adverse effects, such as anaphylaxis and respiratory failure, were similar between the ceftaroline and comparators. ${ }^{47,51}$ The propensity and in vitro frequency of ceftaroline to develop resistance with major pathogens is low. ${ }^{42}$ This was evidenced by multistep resistance studies. ${ }^{52,53}$ Serial passage studies were carried out to determine the probability of developing resistance to ceftaroline in isolates of $S$. pneumoniae, Haemophilus influenzae, Moraxella catarrhalis, MRSA, MSSA, and S. pyogenes. These studies demonstrated low ceftaroline MICs without the devolvement of clones with increased MICs. ${ }^{42,52,53}$ Spontaneous resistance development with vancomycin-resistant $E$. faecalis and vancomycin-susceptible E. faecalis has been demonstrated. ${ }^{33,42}$ Although, not inferior to ceftriaxone in the treatment of CAP patients, ceftaroline has potent activity against community-acquired MRSA as well as difficult to treat bacterial isolates including VISA, VRSA, LRSA, and DNSSA. ${ }^{30}$ Since ceftaroline treats the most common CAP pathogens (including resistant isolates) and possesses an uncomplicated dosing and administration scheme, with a low propensity for drugs interactions, it is a good alternative agent for patients who do not tolerate or respond to other antibacterial therapies. ${ }^{33,42,54}$

\section{Comparative disadvantages}

Currently, ceftaroline has been tested only on specific populations of patients (CAP in patients that require hospitalization, but not ICU care, and complicated skin and skin structure infections). This significantly limits its use, not only for other types of infections, but also for use in patients with pneumonia with different characteristics or risk factors (ie, patients admitted to the ICU or on dialysis). Other disadvantages are that ceftaroline can only be used intravenously. Although intramuscular administration has been studied, it is currently not approved. In addition, there is no oral preparation of ceftaroline that could facilitate transitioning of care. Compared to ceftriaxone, ceftaroline has to be given twice daily instead of once a day. There have been also some reports of the limited stability of ceftaroline after mixing the compound before administration. ${ }^{55}$ Furthermore, there are currently no cost-benefit studies evaluating the use of ceftaroline in the treatment of CAP, but the current cost of this medication is higher than other comparable agents. The cost of ceftaroline is about $\$ 41$ per vial, which corresponds to about $>\$ 80 /$ day. This cost is higher than the comparators available to treat CAP which include intravenous formulations of cephalosporins and respiratory fluoroquinolones. However, if further studies show a benefit in MRSA pneumonia patients, ceftaroline might be a comparable or less expensive alternative compared to vancomycin or linezolid. Caution should be undertaken when using a newly developed medication, but current safety date does not preclude its use as it has a similar safety profile as other cephalosporins.

Even though ceftaroline has exhibited high in vitro potency against MDRSP, MRSA, VISA, VRSA, and DNSSA, and was clinically effective in treating complicated skin-structure and skin infections with theses pathogens, studies on the efficacy in CAP caused by staphylococcal species are lacking. ${ }^{51}$ Until studies are done evaluating other applications of ceftaroline, its use should be limited to the populations where it has shown clinical efficacy.

\section{Future investigations}

The emergence of antimicrobial resistance remains a concern and requires the continued development of novel agents. Given ceftaroline's in vitro success in treating MRSA and other resistant strains of $S$. aureus, future studies should focus on the drug's clinical impact on pneumonias and other infections caused by these pathogens. Additionally, the use of ceftaroline for the treatment of pneumonia should be explored in other populations, including patients admitted to the ICU, as well as those with septic shock, infected with disseminated infections or infections affecting other organs, and requiring renal replacement therapy.

Ceftaroline, similar to other $\beta$-lactam agents, lacks the ability to combat ESBL pathogens. Given ceftaroline's wide coverage of gram-negative and gram-pathogens, there is interest in combining this antibiotic with other agents to provide additional pathogen coverage. Currently, there is an ongoing trail evaluating the combination of ceftaroline with NXL104, an inhibitor of $\beta$-lactamase. Preliminary murine models on NXL104 are promising. ${ }^{56}$ The clinical implications of this drug combination are encouraging in an era of increasing multi-resistant pathogen induced infections.

\section{Conclusion}

Ceftaroline, a novel fifth-generation cephalosporin, is a safe and effective alternative for the treatment of CAP in nonICU hospitalized patients. The drug's in vitro activity has 
exhibited high potency activity against MDRSP, MRSA, VISA, VRSA, and DNSSA; but studies evaluating the clinical outcomes of these effects in CAP are lacking. Although other agents have been recently FDA approved or are under development, further research should continue to evaluate the possible applications of ceftaroline in patients with CAP that require ICU admission and/or have risk factors for MRSA and other resistant gram-positive pathogens that can cause pneumonia. Recent ceftaroline research is focused on evaluating the drug's clinical applications for the treatment of infections due to other pathogens. Studies evaluating the combination of ceftaroline plus NXL104 have demonstrated activity against pathogens, such as multi-resistant gramnegative bacteria. In a time of increasing multi-drug resistant infections, this research may provide additional treatment alternatives to the current antibiotic armamentarium.

\section{Disclosure}

No conflicts of interest were reported by DM, JF, CW, KE, and AN. AA is a consultant and speaker for Forest Laboratories, Bayer Pharma, GlaxoSmithKline, Boehringer-Ingleheim, and Asta-Zeneca. MR participated in advisory boards for OrthoMcNeil-Janssen, Theravan, Forest Laboratories, Johnson and Johnson, and Novartis and has been a speaker for Covidien, BARD Inc, Johnson and Johnson (Ortho-McNeil-Janssen), and Pfizer as well as a consultant for Theravan and Pfizer (Wyeth). MR's time is partially protected by award number K23HL096054 from the National Heart, Lung, and Blood Institute. The content is solely the responsibility of the authors and does not necessarily represent the official views of the National Heart, Lung, and Blood Institute or the National Institutes of Health. The funding agencies had no role in the preparation, review, or approval of the manuscript. The views expressed in this article are those of the authors and do not necessarily represent the views of the Department of Veterans Affairs, nor the University of Texas Health Science Center at San Antonio.

\section{References}

1. Mandell LA, Wunderink RG, Anzueto A, et al. Infectious Diseases Society of America/American Thoracic Society consensus guidelines on the management of community-acquired pneumonia in adults. Clin Infect Dis. 2007;44 Supp1 2:S27-S72.

2. Ferrer M, Menendez R, Amaro R, Torres A. The impact of guidelines on the outcomes of community-acquired and ventilator-associated pneumonia. Clin Chest Med. 2011;32(3):491-505.

3. McCabe C, Kirchner C, Zhang H, Daley J, Fisman DN. Guidelineconcordant therapy and reduced mortality and length of stay in adults with community-acquired pneumonia: playing by the rules. Arch Intern Med. 2009;169(16):1525-1531.
4. Doern GV, Richter SS, Miller A, et al. Antimicrobial resistance among Streptococcus pneumoniae in the United States: have we begun to turn the corner on resistance to certain antimicrobial classes? Clin Infect Dis. 2005;41(2):139-148.

5. Lynch JP III, Zhanel GG. Escalation of antimicrobial resistance among Streptococcus pneumoniae: implications for therapy. Semin Respir Crit Care Med. 2005;26(6):575-616.

6. Lynch JP III, Zhanel GG. Streptococcus pneumoniae: does antimicrobial resistance matter? Semin Respir Crit Care Med. 2009;30(2): 210-238.

7. Pallares R, Liñares J, Vadillo $\mathrm{M}$, et al. Resistance to penicillin and cephalosporin and mortality from severe pneumococcal pneumonia in Barcelona, Spain. N Engl J Med. 1995;333(8):474-480.

8. Tleyjeh IM, Tlaygeh HM, Hejal R, Montori VM, Baddour LM. The impact of penicillin resistance on short-term mortality in hospitalized adults with pneumococcal pneumonia: a systematic review and metaanalysis. Clin Infect Dis. 2006;42(6):788-797.

9. Falcó V, Almirante B, Jordano Q, et al. Influence of penicillin resistance on outcome in adult patients with invasive pneumococcal pneumonia: is penicillin useful against intermediately resistant strains? J Antimicrob Chemother. 2004;54(2):481-488.

10. Aspa J, Rajas O, Rodríguez de Castro F, et al. Drug-resistant pneumococcal pneumonia: clinical relevance and related factors. Clin Infect Dis. 2004;38(6):787-798.

11. Turett GS, Blum S, Fazal BA, Justman JE, Telzak EE. Penicillin resistance and other predictors of mortality in pneumococcal bacteremia in a population with high human immunodeficiency virus seroprevalence. Clin Infect Dis. 1999;29(2):321-327.

12. Metlay JP, Hofmann J, Cetron MS, et al. Impact of penicillin susceptibility on medical outcomes for adult patients with bacteremic pneumococcal pneumonia. Clin Infect Dis. 2000;30(3):520-528.

13. Whitby M, McLaws ML, Berry G. Risk of death from methicillinresistant Staphylococcus aureus bacteraemia: a meta-analysis. Med $J$ Aust. 2001;175(5):264-267.

14. Cosgrove SE, Sakoulas G, Perencevich EN, et al. Comparison of mortality associated with methicillin-resistant and methicillin-susceptible Staphylococcus aureus bacteremia: a meta-analysis. Clin Infect Dis. 2003;36(1):53-59.

15. Taneja C, Haque N, Oster G, et al. Clinical and economic outcomes in patients with community-acquired Staphylococcus aureus pneumonia. J Hosp Med. 2010;5(9):528-534.

16. Boucher HW, Corey GR. Epidemiology of methicillin-resistant Staphylococcus aureus. Clin Infect Dis. 2008;46 Suppl 5: S344-S349.

17. David MZ, Daum RS. Community-associated methicillin-resistant Staphylococcus aureus: epidemiology and clinical consequences of an emerging epidemic. Clin Microbiol Rev. 2010;23(3):616-687.

18. Patel M. Community-associated meticillin-resistant Staphylococcus aureus infections: epidemiology, recognition and management. Drugs. 2009;69(6):693-716.

19. Rosenthal VD, Maki DG, Jamulitrat S, et al. International Nosocomial Infection Control Consortium (INICC) report, data summary for 2003-2008, issued Jun 2009. Am J Infect Control. 2010;38(2): 95-104. e2.

20. Tenover FC, Goering RV. Methicillin-resistant Staphylococcus aureus strain USA300: origin and epidemiology. J Antimicrob Chemother. 2009;64(3):441-446.

21. Labandeira-Rey M, Couzon F, Boisset S, et al. Staphylococcus aureus Panton-Valentine leukocidin causes necrotizing pneumonia. Science. 2007;315(5815):1130-1133.

22. Hota B, Lyles R, Rim J, et al. Predictors of clinical virulence in community-onset methicillin-resistant Staphylococcus aureus infections: the importance of USA300 and pneumonia. Clin Infect Dis. 2011;53(8):757-765.

23. Stryjewski ME, Corey GR. New treatments for methicillinresistant Staphylococcus aureus. Curr Opin Crit Care. 2009;15(5): 403-412. 
24. Villegas-Estrada A, Lee M, Hesek D, Vakulenko SB, Mobashery S. Co-opting the cell wall in fighting methicillin-resistant Staphylococcus aureus: potent inhibition of PBP 2a by two anti-MRSA beta-lactam antibiotics. J Am Chem Soc. 2008;130(29):9212-9213.

25. Kosowska-Shick K, McGhee PL, Appelbaum PC. Affinity of ceftaroline and other beta-lactams for penicillin-binding proteins from Staphylococcus aureus and Streptococcus pneumoniae. Antimicrob Agents Chemother. 2010;54(5):1670-1677.

26. Moisan H, Pruneau M, Malouin F. Binding of ceftaroline to penicillin-binding proteins of Staphylococcus aureus and Streptococcus pneumoniae. J Antimicrob Chemother. 2010;65(4):713-716.

27. Sader HS, Fritsche TR, Kaniga K, Ge Y, Jones RN. Antimicrobial activity and spectrum of PPI-0903M (T-91825), a novel cephalosporin, tested against a worldwide collection of clinical strains. Antimicrob Agents Chemother. 2005;49(8):3501-3512.

28. Ge Y, Biek D, Talbot GH, Sahm DF. In vitro profiling of ceftaroline against a collection of recent bacterial clinical isolates from across the United States. Antimicrob Agents Chemother. 2008;52(9):3398-3407.

29. Brown SD, Traczewski MM. In vitro antimicrobial activity of a new cephalosporin, ceftaroline, and determination of quality control ranges for MIC testing. Antimicrob Agents Chemother. 2009;53(3):1271-1274.

30. Saravolatz LD, Pawlak J, Johnson LB. In vitro activity of oritavancin against community-associated methicillin-resistant Staphylococcus aureus (CA-MRSA), vancomycin-intermediate S. aureus (VISA), vancomycin-resistant S. aureus (VRSA) and daptomycin-nonsusceptible S. aureus (DNSSA). Int J Antimicrob Agents. 2010;36(1): 69-72.

31. Jacobs MR, Good CE, Windau AR, et al. Activity of ceftaroline against recent emerging serotypes of Streptococcus pneumoniae in the United States. Antimicrob Agents Chemother. 2010;54(6):2716-2719.

32. Jones RN, Farrell DJ, Mendes RE, Sader HS. Comparative ceftaroline activity tested against pathogens associated with communityacquired pneumonia: results from an international surveillance study. J Antimicrob Chemother. 2011;66 Suppl 3:iii69-iii80.

33. Kaushik D, Rathi S, Jain A. Ceftaroline: a comprehensive update. Int J Antimicrob Agents. 2011;37(5):389-395.

34. Jones RN, Fritsche TR, Ge Y, Kaniga K, Sader HS. Evaluation of PPI0903M (T91825), a novel cephalosporin: bactericidal activity, effects of modifying in vitro testing parameters and optimization of disc diffusion tests. J Antimicrob Chemother. 2005;56(6):1047-1052.

35. Fenoll A, Aguilar L, Robledo O, et al. In vitro activity of ceftaroline against Streptococcus pneumoniae isolates exhibiting resistance to penicillin, amoxicillin, and cefotaxime. Antimicrob Agents Chemother. 2008;52(11):4209-4210.

36. McGee L, Biek D, GeY, et al. In vitro evaluation of the antimicrobial activity of ceftaroline against cephalosporin-resistant isolates of Streptococcus pneumoniae. Antimicrob Agents Chemother. 2009;53(2):552-556.

37. Croisier-Bertin D, Piroth L, Charles PE, et al. Ceftaroline versus ceftriaxone in a highly penicillin-resistant pneumococcal pneumonia rabbit model using simulated human dosing. Antimicrob Agents Chemother. 2011;55(7):3557-3563.

38. Richter SS, Satola SW, Crispel EK, et al. Detection of Staphylococcus aureus isolates with heterogeneous intermediate-level resistance to vancomycin in the United States. J Clin Microbiol. 2011;49(12): 4203-4207.

39. Steed M, Vidaillac C, Rybak MJ. Evaluation of ceftaroline activity versus daptomycin (DAP) against DAP-nonsusceptible methicillin-resistant Staphylococcus aureus strains in an in vitro pharmacokinetic/pharmacodynamic model. Antimicrob Agents Chemother. 2011;55(7):3522-3226.

40. Steed ME, Rybak MJ. Ceftaroline: a new cephalosporin with activity against resistant gram-positive pathogens. Pharmacotherapy. 2010;30(4):375-389.
41. Zhanel GG, Sniezek G, Schweizer F, et al. Ceftaroline: a novel broadspectrum cephalosporin with activity against meticillin-resistant Staphylococcus aureus. Drugs. 2009;69(7):809-831.

42. Laudano JB. Ceftaroline fosamil: a new broad-spectrum cephalosporin. J Antimicrob Chemother. 2011;66 Suppl 3:iii11-iii18.

43. Kanafani ZA, Corey GR. Ceftaroline: a cephalosporin with expanded gram-positive activity. Future Microbiol. 2009;4(1):25-33.

44. Ge Y, Maynard D, Rickert DE. Comparative pharmacokinetics of ceftaroline in rats, rabbits, and monkeys following a single intravenous or intramuscular injection. Antimicrob Agents Chemother. 2010;54(2):912-924.

45. Jacqueline C, Caillon J, Batard E, et al. Evaluation of the in vivo efficacy of intramuscularly administered ceftaroline fosamil, a novel cephalosporin, against a methicillin-resistant Staphylococcus aureus strain in a rabbit endocarditis model. $J$ Antimicrob Chemother. 2010;65(10):2264-2265.

46. Andes D, Craig WA. Pharmacodynamics of a new cephalosporin, PPI0903 (TAK-599), active against methicillin-resistant Staphylococcus aureus in murine thigh and lung infection models: identification of an in vivo pharmacokinetic-pharmacodynamic target. Antimicrob Agents Chemother. 2006;50(4):1376-1383.

47. File TM Jr, Low DE, Eckburg PB, et al. Integrated analysis of FOCUS 1 and FOCUS 2: randomized, doubled-blinded, multicenter phase 3 trials of the efficacy and safety of ceftaroline fosamil versus ceftriaxone in patients with community-acquired pneumonia. Clin Infect Dis. 2010;51(12):1395-1405.

48. Critchley IA, Eckburg PB, Jandourek A, Biek D, Friedland HD, Thye DA. Review of ceftaroline fosamil microbiology: integrated FOCUS studies. JAntimicrob Chemother. 2011;66 Suppl 3:iii45-iii51.

49. Corrado ML. Integrated safety summary of CANVAS 1 and 2 trials: Phase III, randomized, double-blind studies evaluating ceftaroline fosamil for the treatment of patients with complicated skin and skin structure infections. J Antimicrob Chemother. 2010;65 Suppl 4: iv67-iv71.

50. Panagiotidis G, Bäckström T, Asker-Hagelberg C, Jandourek A, Weintraub A, Nord CE. Effect of ceftaroline on normal human intestinal microflora. Antimicrob Agents Chemother. 2010;54(5): 1811-1814.

51. Corey GR, Wilcox M, Talbot GH, et al. Integrated analysis of CANVAS 1 and 2: Phase 3, multicenter, randomized, double-blind studies to evaluate the safety and efficacy of ceftaroline versus vancomycin plus aztreonam in complicated skin and skin-structure infection. Clin Infect Dis. 2010;51(6):641-650.

52. Clark C, Kosowska-Shick K, McGhee P, et al. Multistep resistance development studies of ceftaroline (CPT) with Haemophilus influenzae and Oraxella catarrhalis. Abstracts of the Fiftieth Interscience Conference on Antimicrobial Agents and Chemotherapy, Boston, MA, 2010. American Society for Microbiology, Washington DC, USA; Poster \#E-814.

53. Clark C, Kosowska-Shick K, McGhee P, et al. Multistep resistance development studies of ceftaroline (CPT) with Streptococcus pneumoniae, Streptococcus pyogenes, staphylococci, and enterococci. Abstracts of the Fiftieth Interscience Conference on Antimicrobial Agents and Chemotherapy, Boston, MA, 2010. American Society for Microbiology, Washington DC, USA; Poster \#E-813.

54. Ishikawa T, NakayamaY, Tomimoto M, et al. Studies on anti-MRSA parenteral cephalosporins. IV. A novel water-soluble N-phosphono type prodrug for parental administration. J Antibiot (Tokyo). 2001;54(4):364-374.

55. Teflaro ${ }^{\mathrm{TM}}$. Package insert. St Louis, Missouri, USA. Forest Pharmaceuticals Inc. Jan 2011.

56. Wiskirchen DE, Crandon JL, Furtado GH, Williams G, Nicolau DP. In vivo efficacy of a human-simulated regimen of ceftaroline combined with NXL104 against extended-spectrum-beta-lactamase (ESBL)producing and non-ESBL-producing Enterobacteriaceae. Antimicrob Agents Chemother. 2011;55(7):3220-3225. 
Infection and Drug Resistance

\section{Publish your work in this journal}

Infection and Drug Resistance is an international, peer-reviewed openaccess journal that focuses on the optimal treatment of infection (bacterial, fungal and viral) and the development and institution of preventive strategies to minimize the development and spread of resistance. The journal is specifically concerned with the epidemiology of antibiotic

\section{Dovepress}

resistance and the mechanisms of resistance development and diffusion in both hospitals and the community. The manuscript management system is completely online and includes a very quick and fair peerreview system, which is all easy to use. Visit http://www.dovepress.com/ testimonials.php to read real quotes from published authors.

Submit your manuscript here: http://www.dovepress.com/infection-and-drug-resistance-journal 